\title{
A study on Association between pregnancy-associated plasma protein-A levels in the first trimester and gestational diabetes mellitus
}

\author{
Lavanya*, T. Sankeerthana, E. Yamini, V. Shilpika, Roopali Jain, Sanober Fatima \\ Bojjam Nashimulu Pharmacy College for Women, \\ e-mail: ID: lavanyasara32@gmail.com
}

\section{ABSTRACT :}

Gestational diabetes is a condition in which a woman without diabetes develops high blood sugar levels during pregnancy. Gestational diabetes generally results in few symptoms however; it does increase the risk of pre-eclampsia, depression, and requiring a Caesarean section. To evaluate whether first-trimester pregnancy associated plasma protein-A multiple of median is associated with gestational diabetes mellitus in pregnant women. This prospectively collected case series was conducted between December 2017 to March 2018 at the obstetric unit Hospital, which is a public tertiary care hospital in Hyderabad. If we screened at-risk women by early OGTT alone, only $6(25.4 \%)$ cases of GDM would be identified. On the other hand, if we subjected atrisk women to early OGTT followed by 28-week OGTT for those who had a normal early OGTT, 12 (49.1\%) cases of GDM would be identified. Since univariate analysis showed that free $\beta$-HCG MoM was not associated with GDM $(P=0.29)$, its confounding effect would be minimal. Thus, an association between GDM and PAPP-A MoM was not detected. Our study showed that with maternal risk factor screening strategies, only 7 (30.2\%) women would undergo OGTT. It showed that the vast majority of women with GDM did not require insulin nor exhibit significant change in PAPP-A MoM during the first trimester. First-trimester PAPP-A MoM was not a useful predictor for development of GDM. A correlation between PAPP-A and HbAlc levels was not observed. Our study showed a high prevalence of GDM at $36.5 \%$, which is higher than that in previous studies.

Key words: pre-eclampsia, depression, Caesarean section

\section{I.INTRODUCTION}

Gestational diabetes is a condition in which a woman without diabetes develops high blood sugar levels during pregnancy. ${ }^{[1]}$ Gestational diabetes generally results in few symptoms ${ }^{[1]}$ however, it does increase the risk of pre-eclampsia, depression, and requiring a Caesarean section. ${ }^{[1]}$ Babies born to mothers with poorly treated gestational diabetes are at increased risk of being too large, having low blood sugar after birth, and jaundice. ${ }^{[2]}$ If untreated, it can also result in a stillbirth. ${ }^{[2]}$ Long term, children are at higher risk of being overweight and developing type 2 diabetes. ${ }^{[2]}$ Gestational diabetes only happens during pregnancy. It means you have high blood sugar levels, but those levels were normal before you were pregnant.

If you have it, you can still have a healthy baby with help from your doctor and by doing simple things to manage your blood sugar, also called blood glucose ${ }^{3}$. After your baby is born, gestational diabetes usually goes away. Gestational diabetes makes you more likely to develop type 2 diabetes, but it won't definitely happen ${ }^{4}$. Gestational diabetes develops during pregnancy (gestation). Like other types of diabetes, gestational diabetes affects how your cells use sugar (glucose). Gestational diabetes causes high blood sugar that can affect your pregnancy and your baby's health ${ }^{5}$.

Any pregnancy complication is concerning, but there's good news. Expectant women can help control gestational diabetes by eating healthy foods, exercising and, if necessary, taking medication. Controlling blood sugar can prevent a difficult birth and keep you and your baby healthy ${ }^{6}$. In gestational diabetes, blood sugar usually returns to normal soon after delivery. But if you've had gestational diabetes, you're at risk for type 2 diabetes. You'll continue working with your health care team to monitor and manage your blood sugar

Gestational diabetes mellitus (sometimes referred to as GDM) is a form of diabetes that occurs during pregnancy ${ }^{7}$. Most women will no longer have diabetes after the baby is born. However, some women will continue to have high blood glucose levels after delivery. It is diagnosed when higher than normal blood glucose levels first 
appear during pregnancy ${ }^{8}$. Gestational diabetes is the fastest growing type of diabetes in Australia, affecting thousands of pregnant women. Between $12 \%$ and $14 \%$ of pregnant women will develop gestational diabetes and this usually occurs around the 24th to 28th week of pregnancy ${ }^{9}$. All pregnant women should be tested for gestational diabetes at 24-28 weeks of pregnancy (except those women who already have diabetes). Women who have risk factors for gestational diabetes should be tested earlier in their pregnancy ${ }^{10}$.

The Aim of the study is to evaluate whether first-trimester pregnancy associated plasma protein-A multiple of median is associated with gestational diabetes mellitus in pregnant women.

\section{EXPERIMENTAL WORK}

This prospectively collected case series was conducted between December 2017 to March 2018 at the obstetric unit Hospital, which is a public tertiary care hospital in Hyderabad. Ethical approval for the study was obtained from the local institutional human research ethics committee.

They were also given dietary and exercise advice and encouraged to perform daily capillary blood glucose monitoring before and 2hours after a meal. If the pre- and post-meal glucose levels frequently exceeded 6.0 and $7.8 \mathrm{mmol} / \mathrm{L}$, respectively, the women were prescribed insulin. All participating women received routine antenatal care until delivery according to our department protocol. The demographic and clinical data were routinely collected by an obstetrician during the first antenatal visit and were entered into the hospital electronic system (antenatal record system). Maternal weight, height, and blood pressure were measured and body mass index (BMI) was calculated. All women who had one or more risk factors for the development of GDM, such as advanced maternal age ( $\geq 35$ years), previous GDM, family history of DM (first-degree relative with DM), a previous macrosomic baby $(\geq 4.0 \mathrm{~kg})$, an unexplained stillbirth, significant glycosuria, or obesity $(\mathrm{BMI} \geq 25 \mathrm{~kg} / \mathrm{m} 2)$ underwent an early 75-g OGTT after the initial visit. The OGTT results were interpreted according to the World Health Organization (WHO) 1999 criteria. ${ }^{11}$ Gestational DM was diagnosed if the fasting blood glucose level was $\geq 7.0 \mathrm{mmol} / \mathrm{L}$ or if the 2-hour OGTT blood glucose level was $\geq 7.8 \mathrm{mmol} / \mathrm{L}$.

Universal first-trimester Down syndrome screening was performed using fetal NT and maternal biochemistry. The ultrasound machine used was the Voluson E8 Expert equipped with a 3-5 MHz convex/ broadband transducer. To determine crown rump length and NT thickness, the protocols outlined by the Fetal Medicine Foundation were followed. The serum levels of free $\beta$-HCG and PAPP-A were measured by the DELFIA Xpress analytical platform. Multiple of median was adjusted for maternal weight and ethnicity. Down syndrome risk was calculated using the Alpha software. All consecutive women with a singleton pregnancy who attended the hospital for their first antenatal visit (before 14 weeks of gestation) during the recruitment period were invited to participate in this study. Written informed consent was obtained from all women who agreed to participate. Women with a multiple pregnancy, pre-existing DM, chronic disease (eg renal disease, hypertension, connective tissue disease), miscarriage, termination of pregnancy, a fetus with a chromosomal or congenital abnormality, or preterm delivery before an oral glucose tolerance test (OGTT) could be performed were excluded. All statistical analyses were performed using PASW Statistics 18, Release Version 18.0.0 (SPSSInc, 2009, Chicago [IL], US). Categorical data were analysed using the Chi squared test or Fisher'sexact test, depending on the data distribution. ${ }^{12}$ For continuous variables with a normal distribution, the independent $t$ test was used. For continuous data with a highly skewed distribution, a non-parametric test (ie Mann-Whitney $U$ test) was used. Sample size was calculated based on two assumptions. First, about $25 \%$ of the population screened will have GDM, according to a previous local study 22 and our departmental annual audit.= Second, there was a $10 \%$ difference in PAPP-A MoM between a GDM and non-GDM group, according to a previous published series.

\section{III.RESULTS AND DISCUSSION}

The study sample In total, 45 women participated in the study of whom 14 (30.2\%) underwent early OGTT. Indications for early OGTT are summarised in Table 1. Overall, GDM was diagnosed in 24 women. Among them, 6 (25.4\%) had an early diagnosis of GDM. All GDM cases were diagnosed based on a 2-hour OGTT blood glucose level of $\geq 7.8 \mathrm{mmol} / \mathrm{L}$; none had a fasting blood glucose level of $\geq 7.0 \mathrm{mmol} / \mathrm{L}$ (Table 2). The remaining 7 women did not develop GDM. The GDM prevalence was 32.5\%. No woman underwent preterm delivery before OGTT. There was no difference in baseline characteristics between those excluded (eg defaulter and decliner) and those 
TABLE 1. Indications for early oral glucose tolerance test

\begin{tabular}{|c|c|}
\hline Indication & No. (\%) of participants \\
\hline Advanced maternal age $(\geq 35$ years) & $24(100.0)$ \\
\hline Obesity (BMI $\geq 25 \mathrm{~kg} / \mathrm{m} 2)$ & $15(62.4)$ \\
\hline Family history of DM* & $17(72.1)$ \\
\hline Previous GDM & $6(26.9)$ \\
\hline Previous macrosomic baby $\dagger$ & $1(2.4)$ \\
\hline Previous unexplained stillbirth & 0 \\
\hline
\end{tabular}

Graph 1. Indications for early oral glucose tolerance test

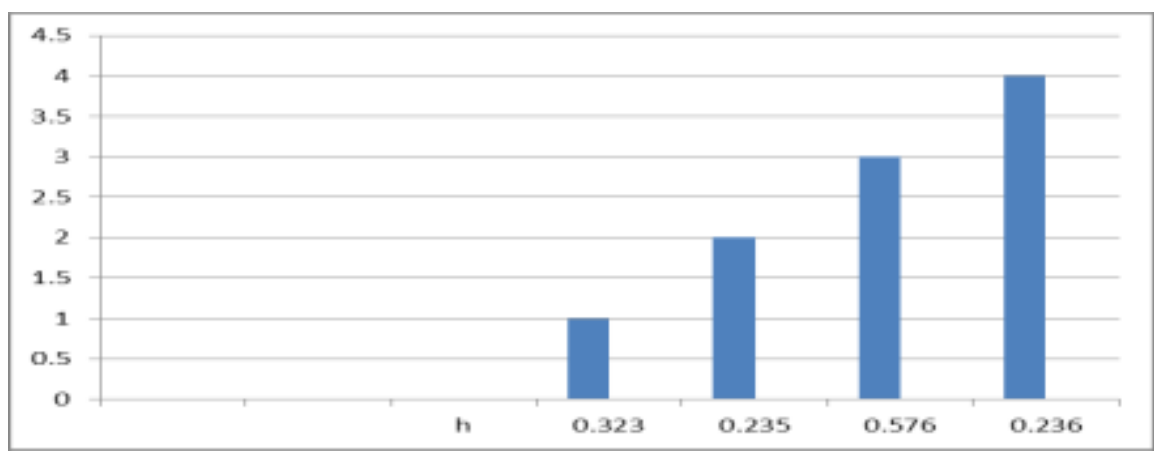

Included in the analysis. The majority $(\mathrm{n}=23,98.8 \%)$ of women with GDM were managed with diet alone. Only two $(1.2 \%)$ required insulin. The maternal characteristics of the women with and without GDM. They were also more likely to have conceived with assisted reproductive technology and to have a family history of DM and a history of GDM. Only two women with GDM required insulin treatment: their PAPP-A MoM was 0.54 and 0.78 , which was low when compared with that of women with GDM who did not require insulin treatment or with the non-GDM group.

TABLE 2. Demographic and clinical characteristics of women with GDM*

\begin{tabular}{|l|l|l|}
\hline Characteristic & GDM $(\mathrm{n}=24)$ & P value \\
\hline Age (years) & $34.3 \pm 31$ & $<0.001$ \\
\hline Advanced maternal age ( $\geq 35$ years) & $(43.2 \%)$ & $<0.001$ \\
\hline Parity & $1(0-1)$ & 0.002 \\
\hline Height $(\mathrm{cm})$ & $160 \pm 156$ & 0.27 \\
\hline BMI $(\mathrm{kg} / \mathrm{m} 2)$ & & \\
\hline Obesity (BMI $\geq 25 \mathrm{~kg} / \mathrm{m} 2)$ & $41(24.3 \%)$ & 0.01 \\
\hline Systolic blood pressure (mm Hg) & $107 \pm 99$ & \\
\hline Diastolic blood pressure (mm Hg) & $63 \pm 59$ & 0.14 \\
\hline Gestational age at USG (weeks) & $12.3 \pm 12.0$ & 0.84 \\
\hline Free $\beta$-HCG (MoM) & $1.05 \pm 0.73$ & 0.29 \\
\hline PAPP-A (MoM) & $0.97 \pm 0.65$ & 0.4 \\
\hline Haemoglobin level (g/L) & $128 \pm 121$ & 0.56 \\
\hline Smoker & 0 & 0.4 \\
\hline ART & $1(4.7 \%)$ & 0.003 \\
\hline Family history of DM $\S$ & $7(30.2 \%)$ & 0.01 \\
\hline Previous GDM & $6(23.7 \%)$ & $<0.001$ \\
\hline Previous macrosomic baby & $1(0.6 \%)$ & 1 \\
\hline Hepatitis B carrier & $1(5.9 \%)$ & 0.98 \\
\hline Alpha-thalassaemia trait & $1(4.1 \%)$ & 0.68 \\
\hline
\end{tabular}




\section{Group 2. Demographic and clinical characteristics of women with GDM}

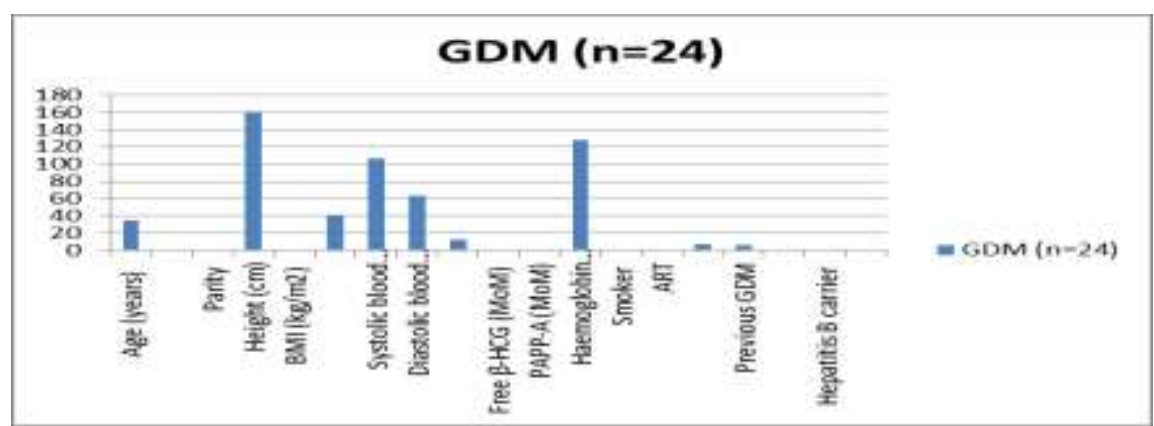

There was a significant but weak correlation between PAPP-A MoM and free $\beta$-HCG MoM $(r=0.2)$.

TABLE 3. Correlations of pregnancy-associated plasma protein-A multiple of median with maternal demographic and clinical characteristics

\begin{tabular}{|l|l|l|}
\hline Maternal characteristic & $\begin{array}{l}\text { PAPP-A } \\
\text { MoM }\end{array}$ & $\begin{array}{l}\text { P } \\
\text { value* }\end{array}$ \\
\hline Age & 0.006 & 0.89 \\
\hline Height & 0.035 & 0.42 \\
\hline Parity & 0.006 & 0.89 \\
\hline Free $\beta$-HCG (MoM) & 0.2 & $<0.001$ \\
\hline Nuchal translucency & 0.056 & 0.21 \\
\hline
\end{tabular}

In this study, 14 (59.2\%) women were nulliparous. Among them, GDM was diagnosed in 24 women. Compared with the non-GDM group, nulliparous women with GDM had no significant change in PAPP-A MoM (median, interquartile range: $0.92,0.63-1.27$ vs $1.0,0.72-1.46 ; \mathrm{P}=0.08$ ). Although univariate analysis showed that the women with and without GDM did not differ significantly in PAPP-A MoM, it is possible that an association between GDM and PAPP-A MoM was obscured by confounding variables. To identify potential confounding variables, Spearman's rho correlation coefficient analysis was performed (Table 4). It revealed that PAPP-A MoM did not correlate with maternal age, height, parity, or NT.

If we screened at-risk women by early OGTT alone, only 6 (25.4\%) cases of GDM would be identified. On the other hand, if we subjected at-risk women to early OGTT followed by 28 -week OGTT for those who had a normal early OGTT, $12(49.1 \%)$ cases of GDM would be identified. Since univariate analysis showed that free $\beta$ HCG MoM was not associated with GDM $(\mathrm{P}=0.29)$, its confounding effect would be minimal. Thus, an association between GDM and PAPP-A MoM was not detected. Our study showed that with maternal risk factor screening strategies, only 7 (30.2\%) women would undergo OGTT.

Although we lacked 1-hour data, we tried to determine whether PAPP-A was associated with GDM based on new diagnostic criteria from the American Diabetes Association (ADA), the International Association of Diabetes and Pregnancy Study Groups (IADPSG), and WHO 2013. A total of 24 women in whom GDM was diagnosed during early pregnancy based on WHO 1999 criteria (but normal by WHO 2013 criteria) did not undergo a second OGTT and were excluded from analysis. The $4(18.5 \%)$ women being identified with GDM. The majority (76\%) of women were diagnosed with GDM based on a 2-hour glucose level of $\geq 8.5 \mathrm{mmol} / \mathrm{L}$. Nevertheless, the GDM group again did not differ to the non-GDM group in terms of PAPP-A MoM

To determine whether PAPP-A MoM and HbA1c levels in women with GDM correlated with each other, Spearman's who correlation coefficient was used. A correlation was not found $(r=0.027 ; \mathrm{P}=0.74)$. The present study showed that women with GDM did not exhibit a significant change in PAPP-A MoM during the first trimester., the studies differed in the selection criteria used to determine when OGTT should be performed, which in turn would target a different study population: some women were tested on the basis of GDM risk factors, some when the women had an abnormal random blood glucose level or 50-mg glucose challenge test, others as a universal screening test as in our study. Second, the studies differed in terms of the OGTT method (75-g 2 hours, 100-g 3 hours, 100-g 2 hours) and diagnostic criteria. 
Tran et al showed that GDM varied substantially in the same population by different diagnostic criteria: 5.9\%ADA, 20.4\% IADPSG, and 24.3\% WHO 1999. Third, the studies may differ in GDM severity, as reflected by the proportion of women with GDM who required insulin treatment. Women with GDM who require insulin may have a more severe type of GDM or undiagnosed pre-existing $\mathrm{DM}^{13}$.

Lovati et al showed that women with GDM had a significantl lower PAPP-A MoM if they received insulin therapy than women with GDM who were managed by diet $(0.56 \text { vs } 0.76 \mathrm{MoM} ; \mathrm{P}<0.001)^{14}$.

Beneventi et al showed a similar result that PAPP-A MoM was significantly lower in GDM managed with insulin treatment than GDM without $(0.87 \text { vs } 1.11 \mathrm{MoM} ; \mathrm{P}=0.031)^{15}$.

These studies showed that insulin-dependent GDMwas more strongly correlated with lower PAPP-A MoM. There were, however, only two (1.2\%) women with GDM in our study who required insulin, a much lower frequency compared with other study populations (12\%-100\%).

The low frequency of insulin treatment in our study population may imply that the majority of affected women had mild GDM and may also explain why our study population did not exhibit changes in PAPP-A MoM during the first trimester, it is known that PAPP-A and free $\beta-\mathrm{HCG}$ are influenced by other maternal or pregnancy variables such as gestational age, ${ }^{11}$ maternal weight, ${ }^{9}$ and smoking. Corrections for these variables were taken into account when calculating the MoM of PAPP-A and free $\beta$-HCG. While different laboratories may have corrected the MoM of PAPP-A and free $\beta$-HCG differently using maternal or pregnancy variables, this may have introduced bias in the assessment of the association between these biochemical markers and GDM. In one study, nonpregnant individuals with type 2 DM were found to have lower PAPP-A levels than non-diabetic controls, PAPPA levels correlated inversely with HbA1c levels $(r=-0.2 ; \mathrm{P}=0.03) .19$ Another study failed to detect this correlation in pregnant women with insulin-dependent DM.17 Similarly, we identified no correlation between PAPP-A and HbA1c levels in women with GDM. These observations suggest that PAPP-A may not be useful for assessing or predicting glycaemic control in women with GDM due to the relatively short duration of women's exposure to GDM that is confined to the latter part of pregnancy.

Our study, which was based on universal screening, showed a high prevalence of GDM (32.5\%). This prevalence was much higher than that of a study conducted by KO Et Al in the early 1990 s $(14.2 \%)^{16}$.

It was also higher than the HAPO study cohort in 2000-2006 that reported a prevalence of GDM in Hong Kong of $14.4 \%$. It is worth investigating this change in the trend of GDM prevalence in Hong Kong that may be due to increasing maternal obesity, adoption of a westernised diet and lifestyle, genetic shift, or other unknown factors. Some authors have proposed that with the new GDM diagnostic criteria, the prevalence of GDM may increase further. This was the first study to assess the association between first-trimester PAPP-A levels and GDM in a population. It showed that the vast majority of women with GDM did not require insulin nor exhibit significant change in PAPP-A MoM during the first trimester. First-trimester PAPP-A MoM was not a useful predictor for development of GDM. A correlation between PAPP-A and HbA1c levels was not observed. Our study showed a high prevalence of GDM at $32.5 \%$, which is higher than that in previous studies.

\section{CONCLUSION}

This was the first study to assess the association between first-trimester PAPP-A levels and GDM in a GDM population. It showed that the vast majority of women with GDM did not require insulin nor exhibit significant change in PAPP-A MoM during the first trimester. First-trimester PAPP-A MoM was not a useful predictor for development of GDM. A correlation between PAPP-A and HbA1c levels was not observed. Our study showed a high prevalence of GDM at $36.5 \%$, which is higher than that in previous studies.

\section{REFERENCES}

1. "Diabetes Blue Circle Symbol". International Diabetes Federation. 17 March 2006. Archived from the original on 5 August 2007.

2. "Gestational Diabetes". NIDDK. September 2014. Archived from the original on 16 August 2016. Retrieved 31 July 2016.

3. Donovan, Peter J; McIntyre, H David (1 October 2010). "Drugs for gestational diabetes". Australian Prescriber. 33 (5): $141-144$.

4. Metzger, B. E.; Coustan, D. R. (1998). "Summary and recommendations of the Fourth International Workshop-Conference on Gestational Diabetes Mellitus. The Organizing Committee". Diabetes Care. 21 Suppl 2: B161-B167. PMID 9704245. And the rest of the issue B1-B167.

5. American Diabetes, A. (2004). "Gestational diabetes mellitus". Diabetes Care. 27 Suppl 1 (Supplement 1): S88-S90.

6. White, P. (1949). "Pregnancy complicating diabetes". The American Journal of Medicine. 7 (5): 609-616.

7. "Archived copy". Archived from the original on 2017-03-02. Retrieved 2017-02-20.

8. Gabbe S.G., Niebyl J.R., Simpson J.L. OBSTETRICS: Normal and Problem Pregnancies. Fourth edition. Churchill Livingstone, 
New York, 2002. ISBN 0-443-06572-1

9. Ross, G. (2006). "Gestational diabetes". Australian family physician. 35 (6): 392-396. PMID 16751853.

10. Chu, S. Y.; Callaghan, W. M.; Kim, S. Y.; Schmid, C. H.; Lau, J.; England, L. J.; Dietz, P. M. (2007). "Maternal Obesity and Risk of Gestational Diabetes Mellitus". Diabetes Care. 30 (8): 2070-2076.

11. Zhang, C.; Bao, W.; Rong, Y.; Yang, H.; Bowers, K.; Yeung, E.; Kiely, M. (2013). "Genetic variants and the risk of gestational diabetes mellitus: A systematic review". Human Reproduction Update. 19 (4): 376-90.

12. Bjorge, T.; Tretli, S.; Engeland, A.; Soule, L. M.; Schisterman, E. F.; Yu, K. F.; Catalano, P. M. (2004). "Relation of Height and Body Mass Index to Renal Cell Carcinoma in Two Million Norwegian Men and Women". American Journal of Epidemiology. 160 (12): 1168-1176.

13. Riskin-Mashiah S, Younes G, Damti A, Auslender R. First-trimester fasting hyperglycemia and adverse pregnancy outcomes. Diabetes Care 2009; 9:1639-43.

14. Lovati E, Beneventi F, Simonetta M, et al. Gestational diabetes mellitus: including serum pregnancy-associated plasma protein-A testing in the clinical management of primiparous women? A case-control study. Diabetes Res Clin Pract 2013; 100:340-7.

15. Beneventi F, Simonetta M, Lovati E, et al. First trimester pregnancy-associated plasma protein-A in pregnancies complicated by subsequent gestational diabetes. Prenat Diagn 2011; 31:523-8.

16. Ko, Sørensen S, Ruge S. Human placental lactogen and pregnancy-associated plasma protein A in first trimester and subsequent fetal growth. Acta Obstet Gynecol Scand 1995; 74:505-8. 